\title{
Routing Protocols for Wireless Sensor Networks: A Survey
}

\author{
Astha Mishra \\ Computer Science Department \\ Lakshmi Narain College of \\ Technology
}

\author{
Sunil Phulre \\ Computer Science Department \\ Lakshmi Narain College of \\ Technology
}

\author{
Vineet Richhariya \\ Computer Science Department \\ Lakshmi Narain College of \\ Technology
}

\begin{abstract}
Contemporary advances in wireless sensor networks have resulted in many new protocols specifically designed for sensor networks where power awareness is most important consideration. Most of the attentiveness, however, has been given to the routing protocols on the grounds that they would differ depending on the applying and network structure. This paper surveys latest routing protocols for sensor networks and presents the principal categories of routing protocols depending upon the key network parameters taken as basis for protocol. The paper provides the basic brief understanding of routing protocol according to their category.
\end{abstract}

\section{Keywords}

Wireless sensor networks (WSN), Routing Protocols.

\section{INTRODUCTION}

Contemporary advances in low power electronic systems and tremendously built-in digital electronics has speed up the progress of micro-sensors. Such sensors are quite often prepared for information processing and data exchange applications. The sensing circuitry measures ambient conditions (the environment surrounding the sensor) and transforms them into an electrical signal. Processing the sort of signal reveals some properties about objects located and/or movements happening within the locality of the sensor. The sensor sends such gathered information, traditionally by way of radio transmitter, to a base station (sink) either immediately or through an information concentration center (a gateway) [1]. The drop in the size and rate of sensors, because of such technological advances, has fueled curiosity within the possible use of enormous set of disposable unattended sensors. Such curiosity has encouraged intensive study in last few years addressing the applicability of collaboration among sensors in data gathering and processing and the coordination and management of the sensing activity and information flow to the sink [1][2]. A normal architecture for such collaborative distributed sensors is a network with wireless links that can be formed among the sensors in an ad hoc manner. Networking unattended sensor nodes are relied upon to have significant effect on the efficiency of numerous military and common applications, for example, battle field reconnaissance, security and disastermanagement [2][3]. These systems process information assembled from different sensors to screen occasions in a territory of investment. Case in point, in a disastermanagement setup, a substantial number of sensors can be dropped by a helicopter. Networking these sensors can help salvage operations by placing survivors, recognizing dangerous regions and making the salvage team more mindful of the general circumstance. Such utilization of sensor networks not just can build the efficiency of salvage operations additionally guarantee the security of the salvage team. On the military side, uses of sensor networks are various. For instance, the utilization of networked set of sensors can constrain the requirement for staff association in the normally hazardous observation missions. What's more, sensor networks can empower a more urban utilization of landmines by making them remotely controllable and target specific keeping in mind the end goal to avert hurting regular people and creatures [5]. Security uses of sensor networks incorporate interruption identification and criminal chasing. On the other hand, sensor nodes are compelled in energy supply and transmission capacity. Such requirements consolidated with a normal arrangement of substantial number of sensor nodes have postured numerous difficulties to the outline and management of sensor networks [5][6]. These difficulties require energy mindfulness at all layers of networking convention stack. The issues identified with physical and connection layers are by and large normal for all sort of sensor applications, in this way the examination on these regions has been centered around system-level force mindfulness, for example, dynamic voltage scaling, radio correspondence equipment, low obligation cycle issues, system parceling, energy-mindful MAC conventions. At the network layer, the primary point is to find routes for energyefficient route setup and dependable handing-off of information from the sensor nodes to the sink so that the lifetime of the network is augmented [7]. Routing in sensor networks is exceptionally difficult because of a few attributes that recognize them from contemporary correspondence and wireless specially appointed networks. As a matter of first importance, it is impractical to construct a worldwide tending to plan for the arrangement of sheer number of sensor nodes. Thusly, traditional IP-based conventions can't be connected to sensor networks. Second, in opposition to regular correspondence networks pretty much all utilizations of sensor networks require the flow of sensed information from numerous areas (sources) to a specific sink. Third, created information trac has significant excess in it since numerous sensors may create same information inside the region of a wonder [4]. Such repetition needs to be abused by the routing conventions to enhance energy and transfer speed usage. Fourth, sensor nodes are firmly obliged regarding transmission power, on-board energy [3][7], preparing limit and capacity and therefore require cautious asset management. Because of such differences, numerous new calculations have been proposed for the issue of routing information in sensor networks. These routing instruments have considered the qualities of sensor nodes alongside the application and structural engineering necessities. Pretty much the majority of the routing conventions can be classified as information driven, progressive or area based albeit there are few different ones taking into account network flow or nature of management (QoS) mindfulness [3][6]. Information driven conventions are question built and depend with respect to the naming of fancied information, which helps in killing numerous excess transmissions. Progressive conventions go for grouping the nodes with the goal that bunch heads can do 
some collection and decrease of information to spare energy. Area based conventions use the position data to hand-off the information to the fancied areas instead of the entire network. The last classification incorporates routing methodologies that are in light of general network-flow displaying and conventions that take a stab at meeting a few QoS necessities alongside the routing capacity. In this paper, we will investigate the routing components for sensor networks grew lately. Every routing convention is examined under the best possible class. Our point is to help better comprehension of the flow routing conventions for wireless sensor networks and bring up open issues that can be liable to further research [9]. The paper is composed as takes after. To be decided of this area, we will briefly abridge the system construction modeling outline issues for sensor networks and their suggestions on information routing. We then set our work separated from former studies on sensor networks. In the Section 2, Routing methodologies are discussed. Section 3 Review onenergy efficient routing protocol. In Section 4, the conclusionof the study is presented with a similar rundown of the studied methodologies and brings up open examination issues.

\section{ROUTING CONSIDERATIONS}

\subsection{System Structure}

Contingent upon the application, different architectures and outline objectives/imperatives have been considered for sensor networks. Since the execution of a routing convention is nearly identified with the building model, in this segment we endeavor to catch structural issues and highlight their suggestions.

\subsection{Network Nodes Properties}

There are three primary parts in a sensor network. These are the sensor nodes, sink and observed occasions. Beside the not very many setups that use portable sensors, a large portion of the network architectures expect that sensor nodes are stationary. Then again, supporting the portability of sinks or group heads (portals) is in some cases regarded essential. Routing messages from or to moving nodes is all the more difficult since route steadiness turns into a critical improvement element, notwithstanding energy, data transmission and so on. The sensed occasion can be either dynamic or static relying upon the application. For example, in a target identification/following application, the occasion (sensation) is alterable while backwoods observing for ahead of schedule fire counteractive action is a case of static occasions. Observing static occasions permits the network to work in a responsive mode, just producing traffic when reporting. Dynamic occasions in many applications oblige intermittent reporting and thus produce significant traffic to be routed to the sink.

\subsection{Node Positioning (Placements)}

An alternate thought is the topological placement of nodes. This is application subordinate and affects the execution of the routing convention. The arrangement is either deterministic or masterminding toward oneself. In deterministic circumstances, the sensors are physically put and information is routed through foreordained ways [6]. However in self arranging systems, the sensor nodes are scattered haphazardly making a framework in an impromptu way. In that framework, the position of the sink or the bunch head is likewise critical regarding energy effciency and execution [8]. At the point when the appropriation of nodes is not uniform, ideal bunching turns into a problem that needs to be addressed to empower energy efficient network operation.

\subsection{Energy Constrains}

Amid the production of a base, the procedure of setting up the routes is significantly influenced by energy contemplations. Since the transmission force of a wireless radio is relative to separation squared or considerably higher request in the vicinity of snags, multi-bounce routing will devour less energy than direct correspondence. Be that as it may, multijump routing presents significant overhead for topology management and medium access control. Direct routing would perform all right if all the nodes were near to the sink [9]. More often than not sensors are scattered arbitrarily over a territory of investment and multi-jump routing gets to be unavoidable.

\subsection{Information ConveyingModels}

Contingent upon the use of the sensor network, the information conveyance model to the sink can be consistent, occasion driven, question determined and half breed. In the ceaseless conveyance demonstrate, every sensor sends information occasionally. In occasion driven and question driven models, the transmission of information is activated when an occasion happens or an inquiry is produced by the sink [8]. A few networks apply a half breed model utilizing a blend of consistent, occasion driven and question driven information conveyance. The routing convention is profoundly influenced by the information conveyance model, particularly concerning the minimization of energy utilization and route soundness [9]. Case in point, it has been finished up in that for an environment checking application where information is constantly transmitted to the sink, a various leveled routing convention is the most effcient option. This is because of the way that such an application produces significant repetitive information that can be totaled on route to the sink, accordingly decreasing traffic and sparing energy.

\subsection{Node Resources}

In a sensor network, different functionalities can be connected with the sensor nodes. In prior works, all sensor nodes are thought to be homogenous, having equivalent limit as far as calculation, correspondence and energy [9]. Be that as it may, contingent upon the application a node can be committed to a specific exceptional capacity, for example, transferring, sensing and accumulation since captivating the three functionalities in the meantime on a node may rapidly deplete the energy of that node [5]. A percentage of the progressive conventions proposed in the writing assign a group head different from the ordinary sensors. While a few networks have picked group heads from the conveyed sensors, in different applications a bunch head is more influential than the sensor nodes regarding energy, data transfer capacity and memory. In such cases, the trouble of transmission to the sink and accumulation is taken care of by the bunch head. Consideration of heterogeneous set of sensors raises numerous specialized issues identified with information routing [7]. For example, a few applications may oblige a different mixture of sensors for observing temperature, weight and mugginess of the encompassing environment, identifying movement through acoustic marks and catching the picture or feature following of moving articles. These unique sensors either conveyed freely or the usefulness can be incorporated on the ordinary sensors to be utilized on interest. Perusing produced from these sensors can be at different rates, subject to different nature of administration imperatives and taking after various information conveyance models, as clarified prior. Accordingly, such a heterogeneous domain makes information routing all the more difficult. 


\subsection{Data Awareness}

Since sensor nodes may create significant repetitive information, comparative bundles from different nodes can be accumulated so the quantity of transmissions would be decreased. Information total is the blend of information from different sources by utilizing capacities [4][6], for example, concealment (dispensing with copies), min, max and normal. Some of these capacities can be performed either part of the way or completely in every sensor node, by permitting sensor nodes to direct in-network information decrease. Perceiving that calculation would be less energy expending than correspondence, significant energy investment funds can be gotten through information conglomeration. This method has been utilized to accomplish energy efficiency and traffic enhancement in various routing conventions. In some network architectures, all total capacities are allotted to all the more compelling and particular nodes. Information conglomeration is additionally doable through sign preparing methods [10]. All things considered, it is alluded as information combination where a node is fit for creating a more exact flag bydiminishing the clamor and utilizing a few procedures, like, beamforming for combinethe signals.

\section{ENERGY-AWARE ROUTING}

These protocols utilize a set of sub-optimal paths at times to build the lifetime of the network. These paths are picked by method for a likelihood capacity, which relies on upon the energy utilization of every path. Network survivability is the principle metric that the methodology is concerned with. The methodology contends that utilizing the base energy path all the time will drain the energy of nodes on that path. Rather, one of the numerous paths is utilized with a certain likelihood so that the entire network lifetime increments. The protocol expect that every node is addressable through a class-based tending to which incorporates the area and sorts of the nodes [5][10].

These protocols use a set ofsub-optimal paths occasionally to increase thelifetime of the network. These paths are chosen bymeans of a probability function, which depends onthe energy consumption of each path. Networksurvivability is the main metric that the approachis concerned with. The approach argues thatusing the minimum energy path all the time willdeplete the energy of nodes on that path. Instead,one of the multiple paths is used with a certainprobability so that the whole network lifetime increases [10][9]. The protocol assumes that each node isaddressable through a class-based addressingwhich includes the location and types of the nodes.

There are three phases of operations in the protocol:

1. Initialization Phase: Localized flooding occurs to find the routes and create the routing tables. While doing this, the total energy cost is calculated in each node. For instance, if the request is sent from node $N_{i}$ to node $N_{j}, N_{j}$ calculates the cost of the path as follows:

$$
C_{N_{j}, N_{i}}=\operatorname{Cost}\left(N_{i}\right)+\operatorname{Metric}\left(N_{j}, N_{i}\right)
$$

Here, the energy metric used computesthe transmission and reception costs along with the residualenergy of the nodes. Paths that have a very highcost are discarded. The node selection is doneaccording to closeness to the destination. Thenode assigns a probability to each of its neighbors in routing (forwarding) table (FT) corresponding to the formed paths. The probability isinversely proportional to the cost, that is:

$$
P_{N_{j}, N_{i}}=\frac{\frac{1}{C_{N_{j}, N_{i}}}}{\sum_{k \in F T_{j}} \frac{1}{C_{N_{j}, N_{k}}}}
$$

$N_{j}$ Then calculates the average cost for reachingthe destination using the neighbors in the forwarding fable $\left(F T_{j}\right)$ using the formula:

$$
\operatorname{Cost}\left(N_{j}\right)=\sum_{k \in F T_{j}} P_{N_{j}, N_{i}} C_{N_{j}, N_{k}}
$$

This average cost for $N_{j}$ is set in the cost field ofthe request and forwarded.

2. Communication Phase: Each node forwards the packet by randomly choosing a nodefrom its forwarding table using the probabilities.

3. Maintenance Phase: Localized flooding isperformed infrequently to keep all the pathsalive.

Therefore, whencompared to traditional routing, it provides anoverall improvement of about $25 \%$ energy saving and a $40 \%$ increase in network lifetime. However, suchsingle path usage hinders the ability of recoveringfrom a node or path failure as opposed to traditional routing. In addition, the approach requiresgathering the location information and setting upthe addressing mechanism for the nodes, whichcomplicate route setup compared to the traditional routing [10][11].

\section{CONCLUSION}

In spite of the fact that the execution of these protocols is guaranteeing as far as energy effciency, further research would be expected to address issues, for example, nature of administration postured by feature and imaging sensors and constant applications. Energy-mindful QoS routing in sensor networks will guarantee ensured data transfer capacity (or delay) through the length of time of association and additionally giving the utilization of most energy efficient path. QoS routing in sensor networks have a few applications including ongoing target following in fight situations, developing occasion activating in checking applications and so on. Right now, there is almost no exploration that takes a gander at taking care of QoS prerequisites in an extremely energy obliged environment like sensor networks. Other conceivable future exploration for routing protocols incorporates the mix of sensor networks with wired networks (i.e. Web). A large portion of the applications in security and ecological observing require the information gathered from the sensor nodes to be transmitted to a server so further investigation could be possible. Then again, the solicitations from the client ought to be made to the sink through Internet. Since the routing necessities of every environment are different, further research is vital for taking care of these sorts of circumstances.

\section{REFERENCES}

[1] SebnemBaydere, YasarSafkan, and OzlemDurmaz. "Lifetime analysis of reliable wireless sensornetworks". IEICE Transactions on Communications 88, 6, 24652472,2006 
[2] Guihai Chen, Chengfa Li, Mao Ye, and Jie Wu.. "An unequal cluster-based routing protocol in wirelesssensor networks". Wireless Networks 15, 2, 193-207. 2009.

[3] Xujin Chen, Xiaodong $\mathrm{Hu}$, and Jianming Zhu. "Minimum data aggregation time problem in wireless sensor networks". In Proceedings of the 1st International Conference on Mobile Ad-hoc and SensorNetworks. Springer-Verlag, Berlin, Heidelberg, 133-142.2005.

[4] K. Martinez, P. Padhy, A. Riddoch, R. Ong, and J. Hart, "GlacialEnvironment Monitoring using Sensor Networks," in Proc. $1{ }^{\text {st }}$ Workshop Real-World Wireless Sensor Netw. (REALWSN), Stockholm,Sweden, 2005, p. 5.

[5] I. Talzi, A. Hasler, S. Gruber, and C. Tschudin, "Permasense:Investigating permafrost with a WSN in the swissalps," in Proc. $4^{\text {th }}$ Workshop Embedded Netw. Sensors (EmNets), Cork, Ireland, 2007, pp.8-12.

[6] S. Tilak, N. B. Abu-Ghazaleh, and W. Heinzelman, "A taxonomy of wireless micro-sensor network models," SIGMOBILE Mob. Comput. Commun. Rev., vol. 6, no. 2, pp. 28-36, 2002.

[7] K. Langendoen, A. Baggio, and O. Visser, "Murphy loves potatoes: experiences from a pilot sensor network deployment in precisionagriculture," in Proc. 20th Int. Symp Parallel Distributed Proc. Symp.(IPDPS), Rhodes Island, Greece, 2006.
[8] R. Hassan, B. Cohanim, and O. de Weck, "A comparison of particleswarm optimization and the genetic algorithm," in Proceedings of theAIAA/ASME/ASCE/AHS/ASC 46th Structures, Structural Dynamics andMaterials Conference, Austin, TX, Apr. 2005.

[9] N. M. A. Latiff, C. C. Tsimenidis, and B. S. Sharif, "Performancecomparison of optimization algorithms for clustering in wireless sensornetworks," in Proceedings of the IEEE International Conference onMobile Ad Hoc and Sensor Systems (MASS), 8-11 Oct. 2007, pp. 1-4.

[10] J. Vesterstrom and R. Thomsen, "A comparative study of differentialevolution, particle swarm optimization, and evolutionary algorithmson numerical benchmark problems," in Proceedings of Congress onEvolutionary Computation (CEC), vol. 2, June 2004.

[11] R. V. Kulkarni, G. K. Venayagamoorthy, and M. X. Cheng, "Bio-inspirednode localization in wireless sensor networks," in Proceedings of theIEEE International Conference on Systems, Man and Cybernetics, SanAntonio, TX, Oct. 2009.

[12] Z. Bojkovic and B. Bakmaz, "A survey on wireless sensor networksdeployment," WSEAS Transactions on Communication, vol. 7, no. 12,pp. 1172-1181, 2008. 